\title{
Desarrollo de la producción oral a través de preguntas y respuestas como técnica de aprendizaje del inglés como lengua extranjera en estudiantes de segundo nivel.
}

\author{
Development of oral production through questions and answers as a \\ technique for learning English as a foreign language in second-level \\ students
}

Ángel Paul Obregón Mayorga. ${ }^{1}$, Mónica Alejandra Logroño Becerra. ${ }^{2}$, Carmita Eulalia Rojas Castro. ${ }^{3}$ \& Gilma Alexandra Gordillo Obregón ${ }^{4}$

\begin{abstract}
.
DOI: https://doi.org/10.33262/concienciadigital.v4i3.1.1820

The aim of this paper was to carry out an action research study to improve the performance of English level 2 students with a basic level of English (A2). For this study the direct method was used i.e., the teacher follows a given pattern of questions and answers during language teaching. The teacher asks questions at a faster pace than a normal conversation to capture the students' attention. The Microsoft Teams platform was used in virtual mode. The 25 students were divided into five groups, for which five channels were created with five students each, the time of each lesson was 20 minutes. Before applying the intervention, the students took a Pre-Test to be evaluated in their oral production, which resulted in a score of 1.69 . The mean of the pronunciation skill was

1 Escuela Superior Politécnica de Chimborazo, Chimborazo, Ecuador, paul.obregon@espoch.edu.ec, https://orcid.org/0000-0001-6831-4848

2 Escuela Superior Politécnica de Chimborazo, Chimborazo, Ecuador, ma_logrono@espoch.edu.ec, https://orcid.org/0000-0002-9348-8601

3 Escuela Superior Politécnica de Chimborazo, Chimborazo, Ecuador, crojasc@espoch.edu.ec, https://orcid.org/0000-0002-8247-0393

4 Universidad Estatal Amazónica, Pastaza, Ecuador, ga.gordilloo@uea.com.ec, https://orcid.org/00000001-7556-6664
\end{abstract}


1.69 points. The mean of the Pronunciation skill was 2.15 , and the mean of the Interactive communication skill was 1.73 which indicated that they are below A2 level. In the posttest the students completed a survey using Likert scale questions to measure the degree of satisfaction in using this direct method of questions and answers, which was coded with the first letters of their full names, obtaining that the mean of the Grammar and Vocabulary skill was 3.08 points, the mean of the Pronunciation skill was 3.24, and the mean of the Interactive communication skill had a value of 3.12, signifying favourable results. With the data obtained, Fisher's exact test was applied using the data from the Grammar and Vocabulary, Pronunciation, Interactive Communication skills of the PreTest and the Post-Test in the experimental group. As a result, the technique based on questions and answers using the direct method significantly improved oral production in English language learning in second level students.

Keywords: Callan method, Oral production, Communicative skills, Questions and answers, Direct method.

\section{Resumen.}

El presente trabajo tuvo como objetivo realizar un estudio de investigación-acción, con el fin de mejorar el desempeño de los estudiantes del segundo nivel de inglés con un nivel básico de inglés (A2). Para este estudio se utilizó el método directo es decir el profesor sigue un patrón dado de preguntas y respuestas durante la enseñanza del idioma. En el que el profesor hace las preguntas a un ritmo más rápido que el de una conversación normal para de esta forma captar la atención de los estudiantes. Se utilizó la plataforma Microsoft Teams en modalidad virtual. Los 25 estudiantes fueron divididos en cinco grupos, para lo que fueron creados cinco canales con cinco estudiantes cada uno, el tiempo de cada lección fue de 20 minutos. Antes de aplicar la intervención los estudiantes tomaron un Pre-Test para ser evaluados en su producción oral que obtuvo como resultados valores de 1.69 puntos. La media de la destreza pronunciación fue de 2.15, y la media de la destreza Interactive communication tuvo un valor de 1.73 lo que indicó que están por debajo del nivel A2. En el Post-Test los estudiantes completaron una encuesta usando preguntas de la escala de Likert para medir el grado de satisfacción al usar este método directo de preguntas y respuestas, el mismo que fue codificado con las primeras letras de sus nombres completos, obteniendo que la media de la destreza Grammar y Vocabulary fue de 3.08 puntos, la media de la destreza de Pronunciación fue de 3.24, Y la media de la destreza Interactive communication tuvo un valor de 3.12, significando resultados favorables. Con los datos obtenidos se aplicó la prueba exacta de Fisher utilizando los datos de las destrezas de Grammar and Vocabulary, Pronunciation, Interactive Communication del Pre-Test y el Post-Test en el grupo experimental. Teniendo como resultado que la técnica basada en preguntas y respuestas utilizando el método directo mejoró notablemente la producción oral en el aprendizaje del idioma inglés en estudiantes del segundo nivel.

Palabras claves: Método callan, Producción oral, Destrezas comunicativas, Preguntas y respuestas, Método directo. 


\section{Introducción.}

La evidente evolución de los diferentes procesos metodológicos en la enseñanzaaprendizaje del idioma inglés como lengua extranjera, ha hecho posible que la comunicación sea cada vez más efectiva. Años atrás, la llegada de los inmigrantes a la isla de Ellis entre 1840 y 1920, trajo consigo muchos desafíos incluyendo el de aprender el idioma para ser entes activos de la nueva sociedad de la cual formarían parte desde aquel entonces (Echeverría, 2021). Quienes arribaron a Ellis desde distintas regiones europeas en búsqueda del sueño americano debieron adaptarse a un nuevo sistema educativo y pese a muchas dificultades, asimilar la lengua inglesa para ser parte de una nueva sociedad. ("Un Viaje Por La Isla De Ellis Y La Isla Del ángel: La Experiencia De Los Inmigrantes," 2018, 237). Hechos como este han sido relevantes en la búsqueda y desarrollo de metodologías que contribuyan al aprendizaje efectivo de una lengua extranjera, ya que se evidencian los primeros rasgos del método directo en la asimilación de vocabulario en contexto a través de oraciones completas. (Cummings, 2014). El proceso enseñanza-aprendizaje del Inglés como lengua extranjera es cada vez más significativo tras haber experimentado varios cambios y reformas que van de la mano con este proceso dinámico que es la comunicación; tomando como punto de partida el método de traducción (1840-1940) cuyas reformas a mediados del siglo 19 sentaron las bases para un nuevo enfoque del aprendizaje de una lengua extranjera de manera natural, lo que más adelante se conocería como método directo cuyos principios marcaron el nacimiento de los "Métodos Era" (1950-1980) (Richards \& Rodgers, 2014, 11-15). Las bases metodológicas del método directo se fundamentan en el aprendizaje de una lengua extranjera a través de la interacción constante y corrección inmediata sin necesidad de recurrir a la traducción en la lengua nativa durante este proceso. Este principio se ha considerado como la clave para el desarrollo de la producción oral en lenguas extranjeras y aunque si bien es cierto, no es el más contemporáneo, ha dado paso a una serie de técnicas y estrategias que hoy en día promueven el uso y dominio del inglés como lengua extranjera por su efectividad al incrementar el grado de motivación y aprendizaje activo de los estudiantes (Netta \& Safura, 2018, 7). La técnica de preguntas y respuestas basada en el método directo tiene un efecto significativo en la fluidez, una característica esencial de la producción oral que consiste en hablar de manera natural con un ritmo que permite mantener la concentración y atención y comunicarse de forma espontánea y coherente. Al implementar esta técnica en clase los estudiantes gradualmente van enriqueciendo su vocabulario y mejorando su pronunciación con la corrección inmediata mientras que los aspectos gramaticales se aprenden a través del uso del idioma en lugar de explicaciones y estudio de reglas. (Ching, 2019, 39)

El aprendizaje del idioma inglés como lengua extranjera en el sistema educativo ecuatoriano involucra el dominio de las cuatro destrezas comunicativas básicas: escuchar, hablar, leer y escribir, sin embargo, las estrategias encaminadas al desarrollo de estas destrezas tienen un alcance limitado ya que muchas veces los logros de aprendizaje son reflejados únicamente en los contenidos gramaticales pero la producción oral es dejada de lado, limitando su fluidez y desarrollo. Una producción oral limitada genera efectos 
negativos en el aprendizaje como inseguridad, temor a cometer errores, respuestas cortas por la falta de práctica, vocabulario limitado, y falta de motivación y confianza entre otros, (Netta \& Safura, 2018, 2).

Considerando este trasfondo, el presente estudio aborda el desarrollo de la producción oral como un componente primordial de las destrezas comunicativas resaltando la importancia del método directo a través de la técnica de preguntas y respuestas para determinar su efectividad en la producción oral en el idioma inglés y ofrecer una respuesta a la interrogante de cómo influye la técnica basada en patrones de preguntas y respuestas establecidos como estrategia para mejorar la producción oral en estudiantes universitarios de segundo nivel de la escuela politécnica superior de Chimborazo a través de una mayor exposición al idioma inglés usando vocabulario en contexto a través de la interacción directa.

\section{Método directo}

El método directo de enseñanza nació en Alemania y Francia alrededor de 1900 y sus creadores fueron Berlitz y Sauze. Se basa en la idea de que el aprendizaje de una 2L tiene que ser una simulación de la lengua materna, ya que es la forma natural en la que las personas adquieren cualquier idioma.

Este método se basa en el aprendizaje del idioma de una manera práctica, sencilla, directa y pedagógica. A diferencia de la metodología practicada en grandes grupos, se concentra en el uso directo del lenguaje, de una manera más práctica y directa y no muy teórica ni generalizada.

En cuanto a su nombre, señala que: "Porque trata de establecer una conexión directa entre la palabra extranjera y la realidad a la que está denominada; es decir, asociar las formas del habla con acciones, objetos, gestos y situaciones, sin la ayuda de la lengua materna. Aquí el docente repite una palabra apuntando al objeto que se denota y lo hace tantas veces como sea necesario hasta que el alumno lo pueda reproducir" (Hernández, 2000).

Los principios del método directo incluyen a estudiantes capaces de obtener un mejor aprendizaje del inglés un papel activo en su enseñanza con el que es concebible que se apropien el idioma, su historia y cultura. Muchos utilizan este método como en Inglaterra en la década de 1960 y utilizado con estudiantes en Londres hace 50 años.

El crecimiento industrial, la globalización y el desarrollo colonial fueron los fundamentos para el nacimiento del método. Según (Hernández, 2000), este método fue capaz de estimular la curiosidad de los aprendices por aprender y progresar. Al parecer, es una conclusión que puede tener poca motivación para los estudiantes a aprender, y encontrar estímulos que hagan crecer esas motivaciones.

Las nuevas tecnologías sumadas al método directo dan la posibilidad de construir el conocimiento de la lengua extranjera a través de diferentes fuentes (plataformas, sistemas, videos, chats, blogs, herramientas de trabajo compartido, redes sociales), permiten 
analizar críticamente la información que obtenemos dibujar. de ellos en conjunto para brindar la capacidad de leer y comprender materiales diversos y dinámicos.

Plataformas como Moodle, Zoom, Microsoft Teams y cualquier recurso tecnológico actual son herramientas adecuadas para mejorar el método directo de aprendizaje del idioma inglés. En cuanto a los chats y correos electrónicos, son una gran oportunidad para que el alumno se comunique con los nativos. (González, 2016).

\section{Definición de Speaking}

La destreza de speaking está considerada como una de las mayores destrezas dentro del proceso comunicativo, especialmente cuando el idioma o lengua nativa o materna está siendo reemplazada por una segunda lengua dentro de este proceso, al igual que escribir se dice se ambas son destrezas activas, ya que permiten que los seres humanos puedan emitir ideas, pensamientos y sentimientos de forma clara y precisa el momento de comunicarse.

Es de vital importancia, hablar de manera efectiva, es decir tener un conocimiento básico de la gramática del idioma, con el fin de estructurar palabras y frases correctas, persiguiendo el propio fin de la comunicación que es emitir una idea clara y coherente de lo que se quiere comunicar, sin distorsionar su significado con la incorrecta pronunciación, generando confusión en el oyente al momento de escuchar.

La destreza de speaking está considerada de primordial dominio en los estudiantes de Inglés como segunda lengua, especialmente en los exámenes internacionales como First certificate, PET, CAE, TOEFL, etc (Universia, 2016) hablar con otras personas es una acto que requiere de una pràctica constante, sobre todo porque se aprende a escuchar con atenciòn y eso es lo que hace que se convierta en un proceso interno que permite adoptar frases y expresiones que nos ayudaràn a aplicarlas en diálogos y conversaciones, tanto formales como coloquiales. Además de que saber hablar en otro idioma, permite desarrollar una gran ventaja comparativa en las organizaciones, puesto que es de vital eficacia poner en práctica todo el proceso de aprendizaje y de esta manera el dominio de esta lengua universal sea útil (Abril, 2019) se enfoca además como una herramienta para el desarrollo de las organizaciones, demostrando la utilidad del uso correcto de la lengua de forma global.

\section{Oral Presentation}

Una presentación oral o hablar en público, es lo que en nuestro medio se conoce comúnmente como exposición, siendo ésta personal o grupal tiene el objetivo de dar a conocer, informar o presentar un argumento o punto de vista de cualquier índole en diversas áreas del conocimiento ya sean sociales, científicas, emocionales, medio ambientales, económicas, etc. Es usual que los estudiantes usen otras técnicas que les ayuden a recordar o enlazar conocimientos y para esto recurren al uso de Powerpoint slides, Prezi o Pecha Kucha que es un acelerado formato de presentación que consiste en un número de slides que se mueven cada 20 segundos (Warwick, 2020). Estas 
presentaciones permiten evaluar no sólo la investigación que realiza cada estudiante o grupo de estudiantes, sino también otro tipo de destrezas de origen cognitivo y transferible. En algunos casos las exposiciones pueden servir como base para el desarrollo de una tarea escrita, generalmente las características que debe tener una exposición se pueden detallar a través de una rúbrica de evaluación, una guía de estudio o un bosquejo de lo que se tomará en cuenta para la evaluación o nota asignada para este trabajo.

\section{Método Callan}

Es un método creado en Italia, en la década del 60 por el lingüista y metodologista Robin Callan, inicialmente fue creado para soldados; se dice que con este método se puede aprender Inglés 4 veces más rápido de lo normal, pero se necesita un nivel de conocimiento del idioma de intermedio a alto, porque no toma en cuenta la gramática per se, y se basa en la repetición, es decir se escucha una palabra o frase y luego se imita, posterior al aprendizaje de las reglas básicas del idioma se aprende a escribir, es decir se enfoca principalmente en el desarrollo del habla o la comunicación. Los libros que se enfocan en enseñar este método contienen una gran cantidad de actividades de refuerzo, ejercicios interactivos y sobre todo preguntas que son hechas por el profesor a lo largo de toda la clase, La idea es que mientras estas preguntas son realizadas el estudiante pueda escuchar la diferencia entre el error y la forma correcta, ya que se cree que se puede aprender de este tipo de corrección desde sus propios errores y ésta corrección permanente nos lleva a un progreso más efectivo, de forma rápida; (Callan Method Organisation, 2021). Es así como cada lección del método Callan contiene una gran variedad de vocabulario y gramática y por lo tanto la estructura del idioma se aprende de manera implícita. Es un método efectivo ya que más de un millón de personas lo han aprendido, incluyendo el nobel de Literatura Gabriel García Márquez.

\section{Microsoft Teams}

Microsoft Teams se define como la plataforma fundada en la nube cuyo objetivo trascendental es el aporte de un grupo de personas en equipo. Su funcionalidad se basa en ser una herramienta de mensajería para empresas, que apoya la comunicación y cooperación en tiempo real entre usuarios dentro y fuera de la organización formada. Es una plataforma principalmente colaborativa donde personas de una misma organización pueden realizar las siguientes actividades:

- Divide y organiza equipos de personas para realizar su trabajo.

- Colabora con información entre miembros

- Hace llamadas o videollamadas entre miembros del grupo.

- Crea archivos de forma colaborativa y con comentarios en vivo, que se acumulan.

- Se une a un equipo más tarde (o ser invitado) y accede a todo el historial de información de ese equipo. (Universidad Complutense, 2020).

"Por supuesto que MS Teams no está reemplazando el tradicional apretón de manos o las reuniones físicas en general, pero es (especialmente en momentos como la pandemia de 
COVID-19) una forma excelente y útil de comunicarse. Además, las características organizativas y muchas otras aplicaciones que colaboran con MS Teams son de gran ayuda para manejar grupos y proyectos con una herramienta central en múltiples carteras, programas y carteras, independientemente de la ubicación, la cultura o la zona horaria. Sin embargo, una limitación de las plataformas digitales, y por lo tanto de MS Teams, es la dependencia de entornos de trabajo y culturas de trabajo de pensamiento moderno". (DTU, 2021).

Moodle

Moodle es un sistema de gestión de aprendizaje en línea gratuito que permite a los educadores crear su propio sitio web privado con cursos dinámicos que amplían el aprendizaje, en cualquier momento y en cualquier lugar (Moodle, 2019). La palabra Moodle

Es un acrónimo de Entorno de aprendizaje dinámico orientado a objetos modulares. También es un verbo en inglés, que habla del proceso de caminar perezosamente deambulando por algo, un juego divertido que a menudo se traduce en creatividad.

Este sistema es una plataforma educativa destinada a brindar a los maestros, gerentes y aprendices un sistema solitario, vigoroso, seguro e integrado para generar una atmósfera de conocimiento personalizado. Una persona podrá transferir el software a sus servidores web o solicitar a uno de los socios expertos de Moodle que le pida ayuda.

Esta plataforma es operada por miles de establecimientos educativos para proporcionar un método organizado y centralizado. Dentro de él, los profesores, estudiantes y administradores de clases pueden crear y dirigir aulas. El chat de mensajería permite la comunicación en un entorno confiable.

\section{Metodologia.}

La población para este estudio fue de 25 estudiantes que se encontraban cursando el segundo nivel de una universidad pública en Ecuador con un nivel básico de inglés de (A2). Para este estudio se utilizó el libro de inglés número 3 de Callan Method que cubre los niveles A1 y A2 según el referente del Marco Común Europeo. Este libro consta de 16 lecciones que se realizaron desde el 28 de mayo hasta el 30 de junio.

Este es un estudio de investigación-acción en el aula, en la cual el investigador-profesor trata de mejorar el desempeño de los estudiantes. Para este estudio se utilizó el método directo ya que el profesor sigue un patrón dado de preguntas y respuestas durante la enseñanza del idioma inglés en la clase. El profesor hace las preguntas a un ritmo más rápido de una conversación normal para de esta forma tener total atención de los estudiantes, esta técnica rápida también impide que los estudiantes traduzcan interiormente las frases a su lengua nativa.

Antes de empezar con las clases los estudiantes tomaron un Pre-Test para ser evaluados en su producción oral antes de aplicar la intervención, este resultado es muy importante 
saber ya que luego será comparado con el resultado de la evaluación del Post-Test. Para el examen de Pre-Test y Post-Test se utilizó el libro 3 de Método Callan y la rúbrica obtenida del libro de Cambridge Key English Test 1 Student's Book que se muestra en la Imagen 1 .

Figura 1

Rúbrica para evaluar la producción oral de estudiantes con nivel A2

\begin{tabular}{|c|c|c|c|}
\hline A2 & Grammar and Vocabulary & Pronunciation & Interactive Communication \\
\hline 5 & $\begin{array}{l}\text { Shows a good degree of } \\
\text { control of simple } \\
\text { grammatical forms. } \\
\text { Uses a range of appropriate } \\
\text { vocabulary when talking } \\
\text { about everyday situations. }\end{array}$ & $\begin{array}{l}\text { Is mostly intelligible, and has } \\
\text { some control of phonological } \\
\text { features at both utterance } \\
\text { and word levels. }\end{array}$ & $\begin{array}{l}\text { - Maintains simple exchanges. } \\
\text { Requires very little prompting } \\
\text { and support. }\end{array}$ \\
\hline 4 & \multicolumn{3}{|c|}{ Performance shares features of Bands 3 and 5 . } \\
\hline 3 & $\begin{array}{l}\text { - Shows sufficient control of } \\
\text { simple grammatical forms. } \\
\text { - Uses appropriate vocabulary } \\
\text { to talk about everyday } \\
\text { situations. } \\
\end{array}$ & $\begin{array}{l}\text { - Is mostly intelligible, despite } \\
\text { limited control of phonological } \\
\text { features. }\end{array}$ & $\begin{array}{l}\text { - Maintains simple exchanges, } \\
\text { despite some difficulty. } \\
\text { - Requires prompting and } \\
\text { support. }\end{array}$ \\
\hline 2 & \multicolumn{3}{|c|}{ Performance shares features of Bands 1 and 3 . } \\
\hline 1 & $\begin{array}{l}\text { - Shows only limited control of } \\
\text { a few grammatical forms. } \\
\text { - Uses a vocabulary of } \\
\text { isolated words and phrases. }\end{array}$ & $\begin{array}{l}\text { - Has very limited control of } \\
\text { phonological features and is } \\
\text { often unintelligible. }\end{array}$ & $\begin{array}{l}\text { - Has considerable difficulty } \\
\text { maintaining simple exchanges. } \\
\text { - Requires additional prompting } \\
\text { and support. }\end{array}$ \\
\hline 0 & \multicolumn{3}{|c|}{ Performance below Band 1 . } \\
\hline
\end{tabular}

Nota. University of Cambridge. (2003). Cambridge Key English Test 1 Student's Book: Examination Papers from the University of Cambridge ESOL Examinations. Cambridge, United Kingdom: Cambridge University Press.

Las clases fueron online usando la plataforma Microsoft Teams. Los 25 estudiantes fueron divididos en cinco grupos de 5 estudiantes cada uno, a estos grupos se los llamó grupo A, B, C, D, E. Para impartir las clases en línea el profesor creó en Microsoft Teams cinco canales con cinco estudiantes cada uno, para así facilitar la enseñanza del idioma inglés con un número óptimo de estudiantes por grupo. El tiempo de la lección para cada grupo fue de 20 minutos.

El rol del profesor fue seguir al pie de la letra el texto escrito en el libro, sin cambiar, aumentar u omitir palabras ya que esto haría que el método utilizado falle. El profesor explicó a los estudiantes el proceso a seguir en su primera lección, de esta manera todos los estudiantes supieron que hacer y en qué orden hacerlo. En este proceso de aprendizaje cada estudiante fue un receptor pasivo que asimilo el conocimiento brindado por profesor siguiendo un esquema programado sistemáticamente, de esta manera por ninguna razón los alumnos pudieron hacer preguntas o interrumpir la clase. Si algún estudiante tuvo alguna pregunta, tenía que esperar con paciencia a que esta pregunta sea aclarada en los temas siguientes o antes de empezar con la siguiente clase.

En cada clase el profesor revisó las seis primeras páginas aprendidas anteriormente por los estudiantes, luego de este proceso, el profesor dio a conocer más vocabulario, durante 
este tiempo el profesor explicó el significado de las nuevas palabras, a veces usando gestos para que los estudiantes puedan entender el significado, durante este proceso los estudiantes no tuvieron que responder a ninguna pregunta, tan solo poner mucha atención. Antes de empezar con la práctica asignada los estudiantes pudieron revisar la lección en su material de aprendizaje por cinco minutos nada más.

Durante el proceso de práctica los estudiantes escucharon con mucha atención a las preguntas del profesor y respondieron inmediatamente usando oraciones completas con la misma estructura que la pregunta. Si algún estudiante no entendió la pregunta, y no supo qué responder, el profesor repitió la pregunta y la respuesta de esta manera el profesor se aseguró de que los estudiantes estén aprendiendo ordenadamente, cada vez que algún estudiante cometía un error al momento de responder una pregunta, el profesor lo corregía inmediatamente haciéndole notar su error.

El dictado fue la única práctica de escritura que tuvieron los estudiantes durante las clases, en esta fase los estudiantes practicaron su recepción auditiva y su producción escrita, la cual fue evaluada por los mismos estudiantes, como tarea los estudiantes tuvieron que escuchar los audios y leer el texto en su material de trabajo.

\section{Resultados.}

Una vez terminada esta investigación los estudiantes fueron evaluados con el Post-Test usando la misma rúbrica utilizada en el Pre-Test. Luego de este examen los estudiantes completaron una encuesta usando preguntas de la escala de Likert para medir el grado de satisfacción al usar este método directo de preguntas y respuestas en el aprendizaje del idioma inglés. Los nombres de los estudiantes fueron codificados usando las primeras letras de sus nombres completos.

El Pre-Test se hizo online y por grupos de 5 estudiantes, a través de la plataforma Microsoft Teams, usando la rúbrica detallada en la Figura 1.

Tabla 1. Puntuación Media del Pre-Test

\begin{tabular}{lllll}
\hline No. & Username & G & P & I \\
\hline 1 & AS & 1 & 2 & 2 \\
2 & AY & 2 & 2 & 2 \\
3 & BC & 2 & 2 & 2 \\
4 & CA & 1 & 2 & 1 \\
5 & CS & 2 & 2 & 2 \\
6 & CV & 2 & 2 & 2 \\
7 & CC & 2 & 2 & 2
\end{tabular}




\begin{tabular}{|c|c|c|c|c|}
\hline 8 & FM & 2 & 3 & 2 \\
\hline 9 & $\mathrm{GC}$ & 1 & 2 & 1 \\
\hline 10 & $\mathrm{GM}$ & 2 & 2 & 2 \\
\hline 11 & GG & 2 & 2 & 2 \\
\hline 12 & GGV & 2 & 2 & 2 \\
\hline 13 & LP & 2 & 3 & 2 \\
\hline 14 & LA & 2 & 2 & 2 \\
\hline 15 & LG & 1 & 2 & 1 \\
\hline 16 & LO & 2 & 2 & 2 \\
\hline 17 & $\mathrm{MM}$ & 2 & 3 & 2 \\
\hline 18 & MG & 1 & 2 & 1 \\
\hline 19 & MGO & 2 & 2 & 2 \\
\hline 20 & MA & 2 & 2 & 2 \\
\hline 21 & OC & 1 & 2 & 1 \\
\hline 22 & PT & 2 & 2 & 2 \\
\hline 23 & PV & 1 & 2 & 1 \\
\hline 24 & QV & 2 & 3 & 2 \\
\hline \multirow[t]{2}{*}{25} & $\mathrm{RV}$ & 2 & 2 & 2 \\
\hline & MEDIA & 1.69 & 2.15 & 1.73 \\
\hline
\end{tabular}

Elaborado por: Grupo de Investigación

Nota. Estos valores fueron obtenidos por el profesor investigador durante la evaluación del Pre-Test
G Grammar and Vocabulary
P pronunciation
I Interactive communication

La Tabla 1, muestra la calificación obtenida por los estudiantes en el Pre-Test, estos valores no satisficieron las expectativas del investigador ya que los estudiantes estuvieron cursando el nivel dos de inglés en una universidad pública de Ecuador y sus calificaciones estuvieron por debajo de este nivel. Como se observa en la tabla 1, los valores de la 
calificación de la destreza Grammar y Vocabulary están muy por debajo del valor máximo de cinco puntos. La media de la destreza Grammar y Vocabulary fue de 1.69 puntos, lo que indica que los estudiantes están por debajo del nivel A2. La Media de la destreza Pronunciación fue de 2.15, este valor también está por debajo del nivel A2. Finalmente, la Media de la destreza Interactive communication tuvo un valor de 1.73 lo que significa que la interacción entre el alumno y el profesor fue muy escasa.

Tabla 2. Puntuación Media del Post_Test

\begin{tabular}{|c|c|c|c|c|}
\hline No. & Username & G & $\mathbf{P}$ & $I$ \\
\hline 1 & AS & 4 & 4 & 3 \\
\hline 2 & AY & 3 & 3 & 2 \\
\hline 3 & $B C$ & 3 & 3 & 3 \\
\hline 4 & $\mathrm{CA}$ & 2 & 3 & 2 \\
\hline 5 & CS & 3 & 3 & 3 \\
\hline 6 & $\mathrm{CV}$ & 4 & 4 & 4 \\
\hline 7 & $\mathrm{CC}$ & 3 & 3 & 3 \\
\hline 8 & FM & 2 & 3 & 2 \\
\hline 9 & $\mathrm{GC}$ & 3 & 4 & 2 \\
\hline 10 & GM & 3 & 3 & 3 \\
\hline 11 & GG & 3 & 3 & 3 \\
\hline 12 & GGV & 3 & 3 & 3 \\
\hline 13 & $\mathrm{LP}$ & 3 & 4 & 4 \\
\hline 14 & LA & 3 & 3 & 3 \\
\hline 15 & LG & 2 & 3 & 3 \\
\hline 16 & LO & 2 & 3 & 3 \\
\hline 17 & $\mathrm{MM}$ & 3 & 3 & 3 \\
\hline 18 & MG & 3 & 3 & 2 \\
\hline 19 & MGO & 3 & 3 & 3 \\
\hline 20 & MA & 3 & 3 & 3 \\
\hline 21 & OC & 3 & 3 & 2 \\
\hline 22 & PT & 3 & 4 & 4 \\
\hline 23 & PV & 2 & 3 & 3 \\
\hline
\end{tabular}




\begin{tabular}{lllll}
24 & QV & 3 & 3 & 3 \\
25 & RV & 4 & 4 & 3 \\
\hline & MEDIA & $\mathbf{3 . 0 8}$ & $\mathbf{3 . 2 4}$ & $\mathbf{3 . 1 2}$ \\
\hline
\end{tabular}

Elaborado por: Grupo de Investigación

Nota. Estos valores fueron obtenidos por el profesor investigador durante la evaluación del Post-Test

La Tabla 2, muestra las calificaciones obtenidas por los estudiantes en el Post-Test, estos valores fueron favorables y satisficieron las expectativas del investigador ya que los estudiantes si mejoraron su nivel de inglés en las tres destrezas. Como se observa en la tabla 2, la Media de la destreza Grammar y Vocabulary fue de 3.08 puntos, la Media de la destreza de la Pronunciación fue de 3.24, Finalmente, la Media de la destreza Interactive communication tuvo un valor de 3.12.

Tabla 3. Comparación del Pre-Test y Post-Test

$\begin{array}{lll}\text { Grammar and Pronunciation } & \text { Interactive } \\ \text { Vocabulary } & & \text { Communication }\end{array}$

\begin{tabular}{|c|c|c|c|c|}
\hline MEDIA & -TEST & 1.72 & 2.16 & 1.76 \\
\hline $\begin{array}{l}\text { MEDIA } \\
\text { TEST }\end{array}$ & POST- & 3.48 & 3.24 & 3.36 \\
\hline
\end{tabular}

Elaborado por: Grupo de Investigación

Nota. Esta tabla muestra una comparación entre los valores de la Media del curso obtenidos en el Pre-test y Post-Test

La Tabla 3. Muestra compara la Media de las calificaciones obtenidas en el Pre-Test y Post-Test. El valor de la Media 3.08 en la destreza de Grammar and Vocabulary en el Post-Test es mayor que la Media 1.69 en el Pre-Test, lo que indica que hay una mejora en esta destreza. Así también el valor de la Media 3.24 en la destreza de la Pronunciation en el Post-Test es mayor que el valor de la Media 2.15 en el Pre-Test. Finalmente, el valor de la Media 3.12 en la destreza de Interactive communication es mayor que el valor de la Media 1.73 en el Pre-Test. 
Figura 2. Comparación de los valores de la Media del Pre-Test y Post-Test

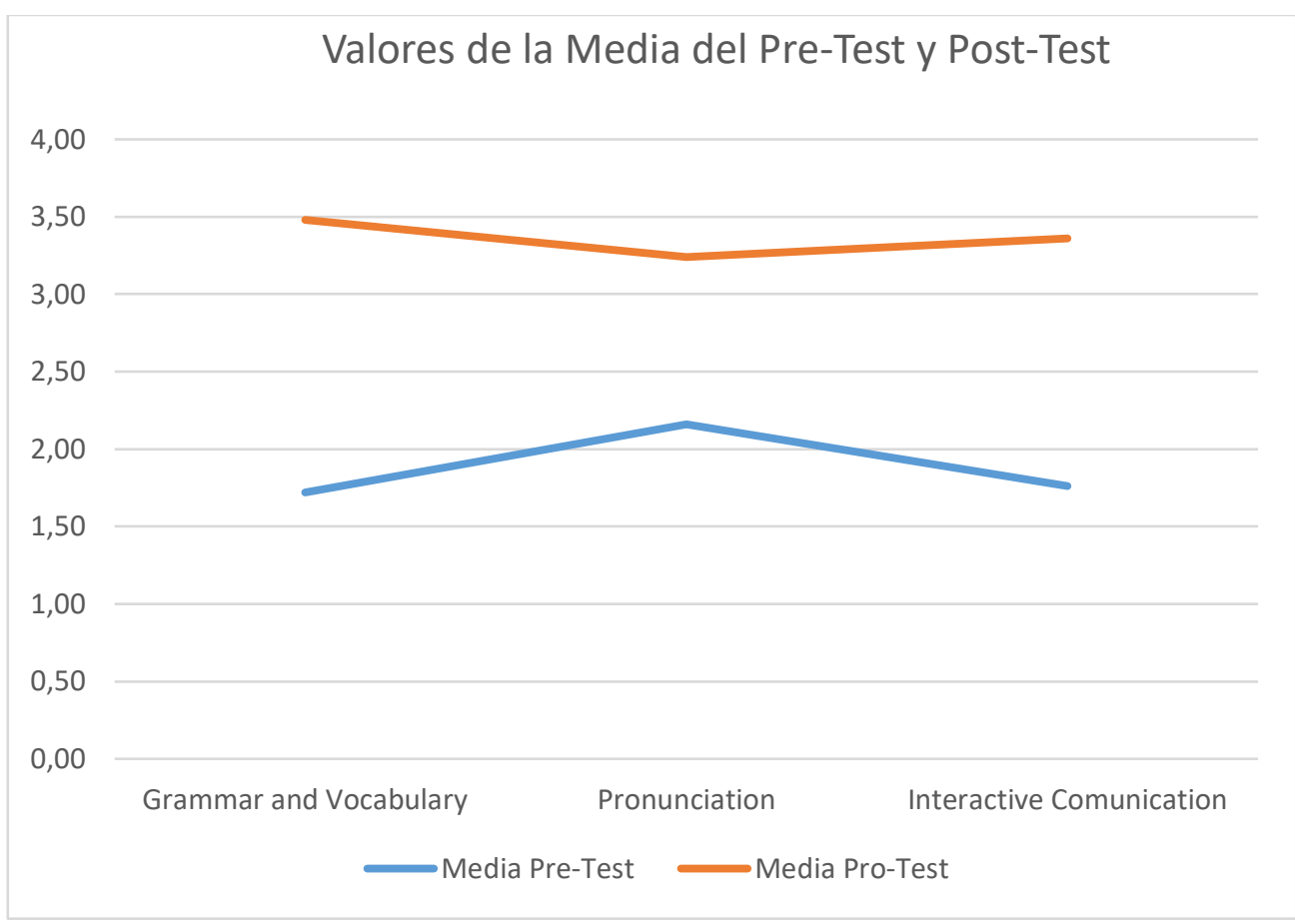

Nota. Los valores del Pre-Test y Post-Test fueron obtenidos por el investigador

Para responder a la pregunta planteada en esta investigación "La técnica basada en preguntas y respuestas utilizando el método directo mejora la producción oral en el aprendizaje del idioma inglés en estudiantes del segundo nivel"

Con los datos obtenidos se aplicó la prueba exacta de Fisher utilizando los datos de las destrezas de Grammar and Vocabulary, Pronunciation, Interactive Communication del Pre-Test y el Post-Test en el grupo experimental.

Tabla 4. F-Test Two-Sample for Variances Grammar and Vocabulary

\begin{tabular}{lll}
\hline & Pre-test (G & Post-test $(G)$ \\
\hline Mean & 1,72 & 3,48 \\
Variance & 0,21 & 0,26 \\
Observations & 25 & 25 \\
df & 24 & 24 \\
& 0,80769230 \\
$\mathrm{~F}$ & 8 \\
& 0,30249346 \\
P(F<=f) one-tail & 6 \\
& 0,50409334 \\
F Critical one-tail & 7 \\
\hline \multicolumn{2}{c}{ Elaborado por: Grupo de Investigación }
\end{tabular}

Nota. Esta tabla muestra el resultado de la prueba de Fisher del Pre-test y Post-Test de la muestra Grammar and Vocabulary. 
Tabla 5. F-Test Two-Sample for Variances Pronunciation

\begin{tabular}{lll}
\hline & Pre-test $(P)$ & Post-test $(P)$ \\
\hline Mean & 2,16 & 3,24 \\
Variance & 0,14 & 0,19 \\
Observations & 25 & 25 \\
df & 24 & 24 \\
$\mathrm{~F}$ & 0,736842105 & \\
P(F<=f) one-tail & 0,22999469 & \\
F Critical one-tail & 0,504093347 & \\
\hline
\end{tabular}

Elaborado por: Grupo de Investigación

Nota. Esta tabla muestra el resultado de la prueba de Fisher del Pre-test y Post-Test de la muestra Pronunciation.

Tabla 6. F-Test Two-Sample for Variances Interactive Communication

\begin{tabular}{lll}
\hline & Pre-test $(I)$ & Post-test (I) \\
\hline Mean & 1,76 & 3,36 \\
Variance & 0,19 & 0,24 \\
Observations & 25 & 25 \\
df & 24 & 24 \\
F & 0,791666667 & \\
P(F<=f) one-tail & 0,285807735 & \\
F Critical one-tail & 0,504093347 & \\
\hline
\end{tabular}

Elaborado por: Grupo de Investigación

Nota. Esta tabla muestra el resultado de la prueba de Fisher del Pre-test y Post-Test de la muestra Interactive communication.

La tabla 4 muestra que la Media (Mean) del Post-Test es mayor a la Media (Mean) del Pre-Test, esto significa que los estudiantes tuvieron un mejor rendimiento después de terminar el estudio. El valor de la varianza en el post-test es de 0.26 y el valor de la varianza en el pre-test es de 0.21 , estos valores nos indican qué tan dispersos están los valores con relación a la Media (Mean). El valor de la observación es de 25 estudiantes, y el valor del grado de libertad (df) es de 24.

El valor de la prueba exacta de Fisher $(\mathrm{F})$ es de 0,807692308, el cual es mayor al valor crítico (F Critical one-tail) de 0,504093347. En la tabla 5 y tabla 6 observamos que el valor de la prueba exacta de Fisher (F) en el Post-Test es mayor al valor de la prueba exacta de Fisher (F) en el Pre-Test. Considerando estos valores rechazamos la hipótesis Nula y cogemos la hipótesis Alterna ya que si existe diferencia entre los valores del Pretest y el Post_Test en el grupo de estudio, lo que demuestra que sí hay mejor rendimiento y se acepta la hipótesis de investigación: "La técnica basada en preguntas y respuestas utilizando el método directo mejora la producción oral en el aprendizaje del idioma inglés en estudiantes del segundo nivel" 
La tabla 7, muestra los resultados obtenidos en la encuesta para determinar el grado de satisfacción en el desarrollo de la producción oral a través de la técnica basada en preguntas y respuestas en el aprendizaje del idioma inglés en los alumnos del segundo nivel.

Tabla 7. Grado de satisfacción en el desarrollo de la producción oral usando preguntas y respuestas en el aprendizaje del idioma inglés.

\begin{tabular}{|c|c|c|c|c|c|}
\hline n. $=25$ & $\begin{array}{l}\text { Totalmente } \\
\text { en } \\
\text { Desacuerdo }\end{array}$ & $\begin{array}{l}\text { En } \\
\text { Desacuerdo }\end{array}$ & $\begin{array}{l}\text { Ni deacuerdo, } \\
\text { ni en } \\
\text { Desacuerdo }\end{array}$ & $\begin{array}{l}\text { De } \\
\text { Acuerdo }\end{array}$ & $\begin{array}{l}\text { Totalmente } \\
\text { de Acuerdo }\end{array}$ \\
\hline $\begin{array}{l}\text { ¿Fue el método } \\
\text { directo de } \\
\text { preguntas y } \\
\text { respuestas útil } \\
\text { en el } \\
\text { aprendizaje del } \\
\text { idioma inglés? }\end{array}$ & $0 \%$ & $4 \%$ & $16 \%$ & $20 \%$ & $60 \%$ \\
\hline $\begin{array}{l}\text { ¿Fue de utilidad } \\
\text { tener el material } \\
\text { de estudio con } \\
\text { las preguntas y } \\
\text { respuestas } \\
\text { durante el } \\
\text { aprendizaje? }\end{array}$ & $0 \%$ & $8 \%$ & $16 \%$ & $24 \%$ & $52 \%$ \\
\hline $\begin{array}{l}\text { ¿Considera } \\
\text { usted que con } \\
\text { este método de } \\
\text { estudio mejoró } \\
\text { su producción } \\
\text { oral? }\end{array}$ & $0 \%$ & $4 \%$ & $20 \%$ & $32 \%$ & $44 \%$ \\
\hline $\begin{array}{l}\text { ¿Fue de utilidad } \\
\text { que el profesor } \\
\text { corrija sus } \\
\text { errores al } \\
\text { momento que } \\
\text { usted está } \\
\text { hablando? }\end{array}$ & $0 \%$ & $4 \%$ & $12 \%$ & $28 \%$ & $56 \%$ \\
\hline
\end{tabular}

Elaborado por: Grupo de Investigación

Nota. Estos valores se obtuvieron de la encuesta de satisfacción realizada a los estudiantes al finalizar el curso. 
Como podemos observar en la tabla 7. la encuesta realizada dio los siguientes resultados: En la primera pregunta, ¿Fue el método directo de preguntas y respuestas útil en el aprendizaje del idioma inglés?, las opciones de Acuerdo y Totalmente de Acuerdo reportaron que el método directo de preguntas y respuestas es muy útil en el aprendizaje del idioma inglés, al sumar estos dos valores dieron un porcentaje de satisfacción $80 \%$, lo que significa que de los 25 estudiantes, 20 estudiantes mostraron completa satisfacción con este método de estudio. En la misma pregunta, el 16\% de estudiantes reportó Ni de Acuerdo ni en Desacuerdo, esto significa que 4 estudiantes mantuvieron una posición neutral con respecto al uso de este método directo de preguntas y respuestas. Un estudiante mostró estar en desacuerdo respecto a esta pregunta lo cual representa el $4 \%$. No hubo ningún reporte en la opción Totalmente en desacuerdo.

En la segunda pregunta, ¿Fue de utilidad tener el material de estudio con las preguntas y respuestas durante el aprendizaje?, las opciones De Acuerdo y Totalmente de Acuerdo reportaron que tener las preguntas y respuestas en el material de clase fue de mucha utilidad para mejorar la producción oral, al sumar estos dos valores dio un porcentaje de $76 \%$, lo que significa que de los 25 estudiantes, 19 estudiantes mostraron que fue muy útil tener las preguntas y respuestas con el audio en el material de estudio. El 16\% de estudiantes indicó estar ni de acuerdo ni en desacuerdo, esto significa que 4 estudiantes mantuvieron una posición neutral con respecto a tener las preguntas y respuestas en el material de clase. Dos estudiantes indicaron estar en desacuerdo, lo cual representa el $8 \%$. No hubo ningún reporte en la opción Totalmente en desacuerdo.

En la tercera pregunta ¿Considera usted que con este método de estudio mejoró su producción oral? Las opciones De Acuerdo y Totalmente de Acuerdo reportaron que este método de preguntas y respuestas mejoró su producción oral después de culminar el curso, al sumar estos dos valores dio un porcentaje de 44\%, lo que significa que de los 25 estudiantes 19 estudiantes mostraron que este método los ayudó a mejorar su producción oral. El 20\% de estudiantes indicó Ni de Acuerdo Ni en Desacuerdo, esto significa que 5 estudiantes mantuvieron una posición neutral con respecto a que si este método mejoró su producción oral. Un estudiante indicó estar en desacuerdo, lo cual representa el $4 \%$. $\%$. No hubo ningún reporte en la opción Totalmente en desacuerdo.

Finalmente, en la cuarta pregunta ¿Fue de utilidad que el profesor corrija sus errores al momento que usted está hablando? Las opciones De Acuerdo y Totalmente de Acuerdo mostraron un porcentaje de $84 \%$, lo que significa que 21 estudiantes reportaron que estaban contentos y satisfechos con que el profesor corrigió sus errores en el momento que estuvieron hablando, de esta manera los estudiantes pudieron revisar en dónde se equivocaron. El 12\% de estudiantes indicó Ni de Acuerdo Ni en Desacuerdo, esto significa que 3 estudiantes mantuvieron una posición neutral a esta pregunta. Un estudiante indicó estar en desacuerdo, lo cual representa el 4\%. No hubo ningún reporte en la opción Totalmente en desacuerdo. 


\section{Discusión}

El uso de la técnica basada en preguntas y respuestas en el aprendizaje del idioma inglés se considera positivo ya que los estudiantes tienen la oportunidad de mejorar su pronunciación y nivel de inglés ya que este método directo sigue un proceso de aprendizaje ordenado en donde el profesor se encarga de hacer las preguntas y el estudiante las respuestas. Este método es eficaz ya que el profesor sigue un patrón de preguntas y respuestas estructuradas que no pueden ser cambiadas y que el estudiante también lo tiene en su material de clase, esto hace que no se pierda el tiempo hablando libremente ya que cuando un estudiante habla libremente solo está utilizando el vocabulario que ya conoce, contrario a este método que tiene una estructura con nuevo vocabulario que se repite durante la clase y de esta manera el estudiante aprende mucho más. También el profesor habla con un ritmo rápido para que los estudiantes pongan atención y no pierdan la concentración.

Este método permite a los estudiantes revisar en casa las lecciones con anterioridad utilizando un audio de las preguntas y respuestas, reforzando así el aprendizaje del idioma inglés. Esta técnica también incluye prácticas de dictado en donde los estudiantes se evalúan a sí mismos.

Este método directo a diferencia de otros no se concentra en la gramática sino en el aprendizaje del idioma en una forma estructurada en donde el estudiante aprende la gramática sin que no note, por su puesto el profesor antes de empezar con la clase responde a preguntas y aclara ciertas dudas con relación a la gramática.

\section{Conclusiones.}

- Este estudio sugiere utilizar este método directo de preguntas y respuestas para mejorar la producción oral en estudiantes de todos los niveles de inglés, ya que es rápido, efectivo, en donde hay una constante práctica de escuchar y hablar durante el transcurso de la clase. Además, el estudiante aprende más porque es un aprendizaje estructurado donde el estudiante se enfoca más en responder correctamente las preguntas sin tener que preocuparse por los errores ya que el profesor repite las respuestas.

- Este método aparte de enfocarse en aprender a escuchar y a hablar se enfoca también en aprender gramática y vocabulario ya que la revisión de las lecciones son sistemáticas asegurándose que los estudiantes recuerden lo que aprendieron durante la clase ya que todos los estudiantes participan respondiendo las preguntas del profesor y no están simplemente de oyentes en una silla.

- Los resultados del post test reflejan que el método directo a través de la técnica basada en preguntas y respuestas mejoró la producción oral de los estudiantes del segundo nivel de inglés, se pudo observar una mayor autonomía y disposición para aprender, además que los resultados de la encuesta de satisfacción realizada por los estudiantes dieron resultados positivos, en donde la gran mayoría de estudiantes estuvieron de acuerdo en que este método mejoró su producción oral. 
- Es posible concluir también que esta técnica se adapta fácilmente a la tecnología ya que el profesor investigador utilizó Microsoft Teams para impartir las clases, dividiendo en canales a los grupos de estudiantes para de esta forma tener un mejor seguimiento de la práctica en cada uno de los estudiantes.

\section{Referencias bibliográficas.}

Abril, C.P.E. (2019). El speaking y su incidencia en los procesos de aprendizaje del idioma Inglés en el centro de Idiomas de la Universidad Técnica de Babahoyo. Universidad Técnica de Babahoyo.

Callan Method Organisation. (2021). Bienvenido al método Callan. https://callan.co.uk/preface/es/

Ching, G. (2019). Teaching English: A Practical Guide for Language Teachers (Primera ed.). Canadian Scholars.

Cummings, L. (2014, Nov 13). Retrieved Julio 24, 2021, from https://www.slideshare.net/lancecummings1/plain-english-and-the-ymcatechnical-writing-classroom-recovering-preprofessional-moments-in-secondlanguage-writing-history

DTU. (2021). Microsoft Teams. http://apppm.man.dtu.dk/index.php/Microsoft_Teams

Echeverría, C. H. (2021, Julio 19). La inmigración en Estados Unidos, una historia de éxito. El Orden Mundial. https://elordenmundial.com/inmigracion-en-estadosunidos-historia/

Gonzales, R. O. (2016). Innovative resources based on ICTs and authentic materials to improve EFL students' communicative needs. In New perspectives on teaching and working with languages in the digital era (pp. 83 -93).

Hernández, F. (2000). Los métodos de enseñanza de lenguas y las teorías de aprendizaje. https://ebuah.uah.es/xmlui/bitstream/handle/10017/950/Los\%20M\%c3\%a9todos $\% 20 \mathrm{de} \% 20$ Ense $\%$ c3\%b1anza\%20de\%20Lenguas\%20y\%201as\%20Teor\%c3\%a das $\% 20$ de $\% 20$ Aprendizaje.pdf?sequence $=1 \&$ isAllowed=y

INTEF. (2021). Moodle, plataforma de aprendizaje. Moodle, plataforma de aprendizaje. http://www.ite.educacion.es/formacion/materiales/184/cd/M2_bloques/tipos_de_ bloques.html

Moodle.

(2019).

Acerca_de_Moodle_FAQ. https://docs.moodle.org/all/es/Acerca_de_Moodle_FAQ

Netta, A., \& Safura, S. (2018, Octubre 17-18). Lecturers' methods in teaching speaking at the university of Muhammadiyah Aceh. Journal of Physics: Conference Series, 1232, 1-7. 10.1088/1742-6596/1232/1/012033 
Richards, J. C., \& Rodgers, T. S. (2014). Approaches ans Methods in LAnguage Teaching (Tercera ed.). Cambridge University Press.

Universia. (2016, Octubre 1). Speaking: estrategias para practicarlo y aprobarlo. https://www.universia.net/ec/actualidad/orientacionacademica/speakingestrategias- practicarlo-aprobarlo-1137521.html

Universidad Complutence. (n.d.). Microsoft Teams: Manual de Uso. Microsoft Teams. https://www.ucm.es/data/cont/media/www/faq/31//TutotialTEAMS_v2_0.pdf

Un Viaje Por La Isla De Ellis Y La Isla Del ángel: La Experiencia De Los inmigrantes. (2018, Julio). DOC.MX, 237. https://xdoc.mx/documents/un-viaje-por-la-isla-deellis-y-la-isla-del-angel-la-experiencia-de-los-5f70109c80002\#

Warwick. (2020, $\quad$ Enero 3$)$ Oral presentations. https://warwick.ac.uk/fac/cross_fac/academicdevelopment/assessmentdesign/methods/3/

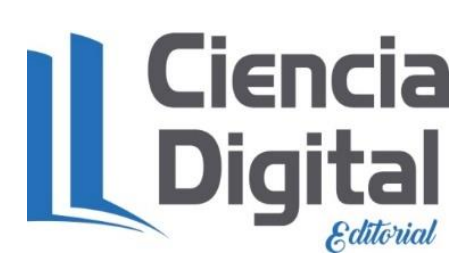




\section{PARA CITAR EL ARTÍCULO INDEXADO.}

Obregón Mayorga, Ángel P., Logroño Becerra, M. A., Rojas Castro, C. E., \& Gordillo Obregón , G. A. (2021). Desarrollo de la producción oral a través de preguntas y respuestas como técnica de aprendizaje del inglés como lengua extranjera en estudiantes de segundo nivel. ConcienciaDigital, 4(3.1), 157-176. https://doi.org/10.33262/concienciadigital.v4i3.1.1820

\section{LCiencia}

El artículo que se publica es de exclusiva responsabilidad de los autores y no necesariamente reflejan el pensamiento de la Revista Conciencia Digital.

El artículo queda en propiedad de la revista y, por tanto, su publicación parcial y/o total en otro medio tiene que ser autorizado por el director de la Revista Conciencia Digital.

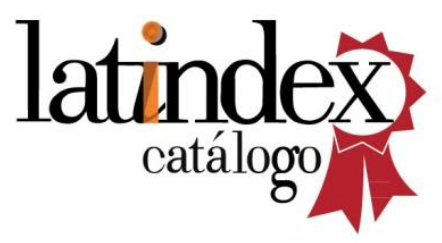

